\title{
Ci sfiliamo (o ci defiliamo?) per un po'
}

\author{
Elisa De Roberto
}

PUBBLICATO: 5 AGOSTO 2021

\section{Quesito:}

Alcuni lettori ci chiedono se l'uso di sfilarsi per defilarsi sia corretto; molti altri domandano quale sia l'ausiliare da usare con il verbo sfilare nel senso di "procedere in fila': "i reparti sono sfilati" o "hanno sfilato"? Infine, qualcuno chiede se sfilamento sia un sinonimo di sfilata.

\section{Ci sfiliamo (o ci defiliamo?) per un po'}

I

1 verbo sfilare, nel significato di 'procedere in fila' e per estensione di 'procedere in corteo', è un verbo intransitivo parasintetico, derivato da fila, col prefisso intensivo $s$ - (da ex-). Come molti altri verbi intransitivi della lingua italiana, può essere usato sia con l'ausiliare avere (i cittadini hanno sfilato davanti al ministero), sia con l'ausiliare essere ( $i$ cittadini sono sfilati davanti al ministero). Entrambi gli usi sono infatti ammessi dai più autorevoli dizionari dell'uso (v. per es. il DISC), anche se in alcune grammatiche scolastiche e nella pubblicistica grammaticale è invalsa una pseudoregola per cui sfilare ${ }^{2}$ nel senso di 'procedere in fila' vorrebbe l'ausiliare essere, mentre avere si userebbe solo con sfilare ${ }^{\mathrm{I}}$, contrario di infilare (e derivato da filo e non da fila): quindi le operaie sono sfilate per il centro, ma le operaie hanno sfilato $i$ lacci dalle scarpe. In realtà tale regola non ha nessun riscontro: sfilare ${ }^{2}$ appartiene a quei verbi intransitivi che presentano oscillazione nella selezione dell'ausiliare (v. Alfonso Leone, Una regola per gli ausiliari, in "Lingua nostra", XXXI, I, I970, pp. 24-30), al pari di correre o volare (o prevalere, come ha osservato un lettore). Ricorrendo alla terminologia linguistica potremmo dire che sfilare, verbo di base inergativo, può anche comportarsi come inaccusativo. La lettura inergativa si determina quando il soggetto del verbo si comporta come il soggetto dei verbi transitivi e il processo espresso del verbo esprime una certa intenzionalità; quando il verbo è inaccusativo invece il soggetto si comporta come un oggetto diretto, presentando dunque un minor coinvolgimento intenzionale nell'azione e un più basso grado di agentività. I verbi correre e volare illustrano alla perfezione questo diverso comportamento: si pensi alla distinzione tra la frase ho corso per quattro chilometri o ho volato per l'Alitalia (valore inergativo), rispetto a sono corso a casa o sono volato a Parigi con il volo delle sei (valore inaccusativo), dove si prospetta non tanto l'azione di correre o volare in sé, quanto uno spostamento. Anche persfilare si potrebbe ipotizzare un'analoga distribuzione degli ausiliari: si valuti la diversa accettabilità di ${ }^{?} g l i$ operai sono sfilati rispetto a gli operai sono sfilati per le vie del centro. La seconda formulazione sembra più accettabile, mentre nel primo caso sarebbe più naturale dire gli operai hanno sflato. Di contro, in alcuni contesti è preferibile l'uso di essere:

- quando a sfilare sono oggetti inanimati:

[Kim Jong-Un] ha esibito il suo sorriso trionfante quando nella piazza dedicata al nonno Kim Il Sung sono sfilati nuovi missili di dimensioni impressionanti. ("Corriere della Sera", Io/ro/2020)

- quando il verbo è impiegato in contesti figurati:

Al momento dellincidente l'intera sua vita gli è sfilata davanti. 
Quando invece si impiega sfilare nel senso di 'presentare una collezione di moda' è normale l'uso di avere, specialmente se il soggetto è lo stilista/il creatore della collezione (e non i modelli che concretamente percorrono la passerella):

Appena laureata presso il College of Arts di Edimburgo, [Alice Firman] ha sfilato con la sua collezione PA'RÒLE. (“Cosmopolitan", 28/II/2019)

Nonostante ciò, restano ampie zone di sovrapponibilità fra i due ausiliari, anche se negli ultimi decenni si registra una tendenza piuttosto accentuata dellitaliano contemporaneo a selezionare il verbo avere (Federica Venier, Da 'essere' ad 'avere': una tendenza dell'ausiliare in italiano, in "Annali dell'Università per Stranieri di Perugia", Nuova Serie, VI, 25, I998, pp. 5I-69).

È invece generalizzato l'uso di essere nella formazione dei tempi composti della forma pronominale intransitiva sfilarsi, quando impiegata nell'accezione di 'andarsene, allontanarsi da un luogo uno dopo l'altro o alla chetichella' (GDLI, s.v. sfilare ${ }^{2}$ ). In tale accezione il verbo ricorre già nell'italiano medievale, come mostra la precoce occorrenza della Cronica di Matteo Villani:

per la qual cosa li usciti guelfi soprastati al termine più di due dì, non avendo novelle che venissono, si cominciarono a sfilare (Matteo Villani, Cronica. Con la continuazione di Filippo Villani, a cura di Giuseppe Porta, 2 voll., Parma, Fondazione Pietro Bembo / Ugo Guanda Editore, 1995, V, 64, p. 688)

ma non è estraneo alla lingua novecentesca, come evidenza l'attestazione fenogliana:

Si sfilarono tutti, in punta di piedi e a respiro mozzo. (Beppe Fenoglio, Il partigiano Johnny, in Romanzi e racconti, a cura di Dante Isella, Torino, Einaudi, 200I, p. 616)

Il verbo sfilarsi può essere impiegato negli stessi contesti e con un significato prossimo a quello di defilarsi:

I renziani si sono defilati anche sull'emendamento per rinviare le norme di equiparazione dei partiti alle fondazioni. (www.ilprimatonazionale.it, o2/o2/2019)

I renziani si sono sfilati da tempo, anche se non rinunciano ad usare la tattica del dondolio fra aperture e chiusure assolute. ("Il Manifesto", 06/08/2020)

Con ciò non si intende dire che sfilarsi è un sinonimo di defilare (possiamo occupare una posizione defilata, ma non una posizione sfilata, ad esempio), ma che per esprimere l'idea di qualcuno che voglia uscire da una situazione poco gradita entrambi i verbi possano essere usati.

Il verbo defilarsi viene da se défiler, che in francese ha la stessa etimologia di sfilarsi (anche se il prefisso è diverso) e lo stesso nucleo semantico principale (défilée infatti vuol dire 'sfilata'). Defilar(si) è entrato nel vocabolario militare italiano, sul finire del Settecento, con il significato molto specifico di 'proteggere qualcosa o proteggersi dall'osservazione e dal tiro del nemico', poi generalizzatosi nel senso di 'nascondersi, scansare un compito non gradito'.

Non sono dunque scorrette formulazioni come Di Stefano, ministro Di Maio non si è sfilato dal dibattito su riconoscimento Palestina ("Reset", $18 / 02 / 2020$ ) o Gli altri soggetti che avevano avanzato manifestazioni dinteresse (ventisei, aveva annunciato Nicastro il 10 maggio scorso) si sono sfilati da tempo (Reuters.com, 20/07/20r6). La convergenza semantica fra i due verbi dipende del resto dal fatto che in contesti di questo tipo sia defilarsi sia sfilarsi sono impiegati in maniera figurata. 
L'atteggiamento censorio che alcuni parlanti mostrano verso frasi di questo tipo è da ricondurre a vari fattori: alla ricerca di un'avanzata specializzazione degli usi lessicali (soprattutto nello scritto);

alla particolare storia dei due verbi (nel corso dell'Ottocento defilare ha avuto maggior fortuna del concorrente "autoctono" come tecnicismo militare); alla tendenza a riconoscere nei prefissati con destrutture più colte e ricercate rispetto ai prefissati con $s$ -

Non andrà inoltre sottovalutato il fatto che molti dizionari dell'uso non riportano tra i significati di sfilarsi, quello di 'andarsene alla chetichella', che invece ritroviamo nel dizionario storico della lingua italiana (GDLI) e nel Nuovo De Mauro. Qui al verbo sfilarsi' 'andarsene, allontanarsi da un luogo uno dopo l'altro o alla chetichella' è attribuita la marca d'uso BU, di basso uso, che individua i vocaboli rari, tuttavia circolanti ancora con qualche frequenza in testi e discorsi del Novecento. Certamente gli usi appena visti, molto frequenti nella cronaca politica, concorrono a rivitalizzare sfilarsi e a diffonderne il significato di 'sottrarsi da una situazione sgradita'.

Rispetto all'oscillazione tra sfilata e sfilamento segnalata da vari lettori, va precisato che l'opzione più corrente e usuale per indicare l'azione di sfilare è sfilata, nominale deverbale femminile formato a partire dal participio passato sfilato: la sfilata della collezione autunno/inverno, la sfilata delle forze armate, ecc. Il termine sfilamento (nome di azione derivato dal medesimo verbo, da non confondere con sfilamento derivato di sfilare nell'accezione di 'estrazione di un oggetto inserito o infilato') è invece più specifico, in quanto fa riferimento al solo ambito militare, dove è sinonimo di rassegna, rivista, sfilata (GDLI, s.v. sfilamento ${ }^{2}$ ), anche se usato molto più raramente.

\section{Cita come:}

Elisa De Roberto, Ci sfiliamo (o ci defiliamo?) per un po', "Italiano digitale", XVIII, 2021/3 (lugliosettembre)

DOI: $10.35948 / 2532-9006 / 2021.10594$

Copyright 2021 Accademia della Crusca

Pubblicato con licenza creative commons CC BY-NC-ND 